\title{
Avaliação da qualidade da prescrição de medicamentos de um hospital de ensino
}

\author{
Evaluation of quality medication prescription of a teaching hospital
}

Patrícia Taveira de Brito A raújo ${ }^{1}$

Severina Alice Costa U chôa ${ }^{2}$

${ }^{1} \mathrm{H}$ ospital de Pediatria Prof. Heriberto Ferreira Bezerra, UniversidadeFederal do

Rio Grande do Norte. Av.

Gal. Gustavo Cordeiro de

Farias s/n, Petrópolis.

59012-170 Natal RN.

patbrito@ufrnet.br

${ }^{2} \mathrm{Núcleo} \mathrm{deEstudos} \mathrm{em}$

Saúde Coletiva,

UniversidadeFederal do

Rio Grande do Norte.
Abstract The errors from doctor prescriptions can cause damage to the patient's health, consequently it is necessary to identify and to prevent them. This work aimed to evaluate if the legal and institutional aspects that are present in doctor prescription at the public and university pe diatric hospital to make a diagnosis from the situation, and then to correct the problems. A survey was made was made using a cross-sectional method, where copies of 1,590 prescriptions were studied after the U niversity Committee of Research approved the survey. The average was 4,47 drugs per prescription and following data were detectable: readable $32.39 \%$ of the prescriptions were unreadable, $49.81 \%$ presented only the commercial name, $5.25 \%$ of the drugs were not standardized. Quality of prescription in the chosen hospital needs to be better to avoid medication errors and the health care process gets safer. When prescription is unreadable, they can confuse health professionals and damage patients.

Key words Prescribing, M edication errors, Readable
Resumo Os erros provenientes das prescrições médicas podem provocar sérios danos à saúde dos pacientes; por isso, é imprescindível que sejam identificados e prevenidos. 0 objetivo deste trabalho foi avaliar a presença dos requisitos legaise institucionais da prescrição médica de um hospital pediátrico público de ensino, a fim de se obter um diagnóstico da situação, para então serem aplicadas as medidas corretivas. A partir da autorização do comitê de pesquisa, foi feito um estudo observacional do tipo transversal, quantitativo, no qual foram analisadas, durante três meses, todas as segundas vias das prescrições dos pacientes hospitalizados. Foram analisadas 1.590 prescrições, cada uma tendo em média 4,47 medicamentos, e obtiveram-se os seguintes dados: quanto à legibilidade, 32,39\% das prescrições eram pouco legíveis, 49,81\% continham medicamentos prescritos por nome comercial, 5,25\% dos medicamentos prescritos não eram padronizados. A qualidade da prescrição do hospital em estudo precisa ser melhorada, a fim de que sejam evitados erros demedicação eo processo deassistência à saúdese torne mais seguro. A falta de clareza nas prescrições pode confundir os profissionais de saúde e causar danos ao paciente.

Palavras-chave Prescrição, Erros de medicação, Legibilidade 


\section{Introdução}

As farmácias têm como uma das funções primordiais a dispensação dos medicamentos de acordo com a prescrição médica, nas quantidades e especificações solicitadas, de forma segura e no prazo requerido, promovendo o uso seguro e correto de medicamentos e correlatos ${ }^{1}$. No Brasil, as farmácias hospitalares têm evoluído e se organizado com o objetivo principal decontribuir para aqualidade da assistência à saúde. Tendo o medicamento como instrumento para o pleno exercício dessa função, elas devem ter como foco de atenção o paciente e as necessidades deste. Assim, sua organização e sua prática devem prevenir queerros relacionados aos medicamentos ocorram. Dentre os processos que envolvem o ciclo do medicamento está o da prescrição e é nele que se deterá a pesquisa, procurando identificar a qualidade das prescrições do hospital em estudo quanto aos requisitos legais e institucionais.

O hospital em estudo éum hospital integrante da Rede Sentinela (ANVISA) e é um hospital de ensino, o qual tem papel primordial na formação dos profissionais de saúde e, por consequência, no cenário da assistência à saúde, o que justifica a necessidade deidentificar a qualidade das prescrições produzidas nessa instituição, a fim de que os erros possam ser corrigidos, melhorando a qualidade da assistência ao paciente?2.

A prescrição medicamentosa éum documento legal pelo qual se responsabilizam quem prescreve (médico) equem dispensa o medicamento (farmacêutico), estando sujeito à legislação de controle e vigilância sanitários. No âmbito hospitalar, a prescrição de medicamento inclui mais um componente, queéa equipe de enfermagem, a qual administra o medicamento ${ }^{3}$. Os erros provenientes das prescrições médicas podem provocar sérios danos à saúde dos pacientes; por isso, éimprescindível queos erros sejam identificados e prevenidos ${ }^{4}$.

\section{Objetivo}

A pesquisa teve por objetivo avaliar a presença dos aspectos legais e institucionais da prescrição médica de um hospital público de ensino e pediátrico, a fim de que se tivesse um diagnóstico da situação para então serem aplicadas as medidas corretivas.

Os objetivos específicos foram identificar se as prescrições estão legíveis ou não; identificar se as prescrições estão contemplando os dados do paciente; identificar se as prescrições estão contemplando dados sobre o medicamento e identificar o número de medicamentos prescritos fora da padronização.

\section{Uso racional de medicamentos}

Uma das preocupações da Organização M undial de Saúde é a disseminação da utilização racional dosmedicamentos. Seguindo essa orientação, foi elaborada a Política Nacional de M edicamentos, que tem como uma de suas diretrizes e prioridades a promoção do "Uso Racional de Medicamentos" (URM ) 4 . 0 URM é definido como 0 processo que compreende a prescrição apropriada, a disponibilidade oportuna e a preços acessíveis, a dispensação em condições adequadas, 0 consumo nas doses indicadas, nos intervalos definidos, no período de tempo indicado, de medicamentos eficazes, seguros e de qualidade. Pela definição de URM, portanto, a prescrição apropriada éum componente importante para a utilização adequada do medicamento; no entanto, no Brasil, ainda há poucas informações sobre a qualidade das prescrições 5 .

Para a O rganização M undial da Saúde, a farmacovigilância compreende a ciência e as atividades relativas à identificação, avaliação, compreensão e preven ção de efeitos adversos ou qualquer problema possível relacionado com fárma$\cos ^{6}$. Esse campo deatividadetem se expandindo e, recentemente, incluiu novos elementos de observação e estudo, embora com um atraso injustificado, uma vez que em 1995 já se chegara à formulação de uma proposta e de estratégias bastante consistentes para institucionalizar um sistema de farmacovigilância em nível nacional ${ }^{7}$.

Os erros de medicação constituem um aspecto de importância dentro da assi stência à saúde, quando se pensa em promover o uso racional de medicamentos. 0 Instituto de M edicina Americano informa que entre 44.000 e 98.000 americanos morrem anualmente em decorrência de erros na medicação eque esses erros ocorrem em $2 \%$ a $14 \%$ dos pacientes hospitalizados 8 . Dentre os erros de medicação, estão os relacionados à prescrição. Alguns trabalhos têm demonstrado que esses erros estão presentes nas unidades hospitalares e podem causar danos à saúde; sendo assim, faz-se necessário identificálos e criar estratégias para preveni- $\operatorname{los}^{9-14}$.

A National Coordinating Council for M edication Error Reporting and Prevention (NCCM ERP) define erro de medicação "como qual- 
quer evento que possa ser evitado e que, de fato ou potencialmente, leve ao uso inadequado de medicamento". Isso significa que o uso inadequado pode ou não lesar o paciente, não importando se o medicamento está sob o controle de profissionais de saúde, do paciente ou do consumidor ${ }^{15}$. Em entrevista à revista Pharmacia Brasileira no ano de 2005, M ário Borges Rosa, farmacêutico mineiro, estudioso de erros de medicação, disse que tanto no curso de graduação como no exercício profissional dentro das unidades de saúde, a abordagem atualmente utilizada deve ser totalmente modificada 5 .

Os eventos adversos preveníveis e potenciais relacionados a medicamentos são produzidos por erros de medicação e a possibilidade de prevenção é uma das diferenças marcantes entre as reações adversas e os erros de medicação. A reação adversa a medicamentos éconsiderada como evento inevitável, ainda que se conheça a sua possibilidade de ocorrência, e os erros de medicação são, por definição, preveníveis. Decorredisso que, com ações bem planejadas, é possível prevenir os erros, melhorando a qualidade da assistência prestada nas unidades de saúde ${ }^{16}$.

O erro pode estar relacionado à prática profissional, a produtos usados na área da saúde, procedimentos, problemas de comunicação “ incluindo-se prescrição, rótulo, embalagem, nome, preparação, dispensação, distribuição, administração, educação, monitoramento e uso de medicamentos ${ }^{17}$.

Dentre os erros de medicação, está o de prescrição, o qual contribui significativamente para o índicetotal dos erros demedicação etem elevado potencial para resultar em consequências maléficas para o paciente. Estima-seque, em cada dez pacientes admitidos no hospital, um esteja em risco potencial ou efetivo de erro na medicação $0^{18}$. Esse risco aumenta quando os profissionais não conseguem ler corretamente as prescrições, devido à letra ilegível ou à falta de informações necessárias para a correta administração, como via, frequência, etc. ${ }^{11,12}$.

No ambiente hospitalar, o primeiro passo para prevenir os erros de medicação e aumentar a segurança para os pacientes envolve, necessariamente, a prescrição demedicamentos, já queessa éa primeira etapa do circuito dos medicamentos no hospital ${ }^{19}$.

Como parte desse processo, a Agência N acional de Vigilância Sanitária (ANVISA) tem promovido cursos para a formação de multiplicadores, para o uso da farmacoterapia baseada em problemas, entre outras ações, tais como a cria- ção do Comitê de U so Racional de M edicamentos, a parceria estabelecida com entidades deprescritores edispensadores, a realização de dois congressos de uso racional e a participação da Rede de Hospitais Sentinelas, que teve esse tema nos projetos de $2007^{5,20}$.

No âmbito da farmacovigilância, os hospitais sentinelas representam uma estratégia adicional para integrar a monitoração de medicamentos à prática clínica. 0 envolvimento dos profissionais de saúde com os princípios da farmacovigilância tem grande impacto na qualidade da assistência, tanto nos aspectos relacionados à prescrição, influindo diretamente na prática médica, quanto na dispensação e no uso de medicamentos: 0 médico passa a agregar novas referências à avaliação do tratamento a ser prescrito; os enfermeiros aprendem a relacionar eventos da prática de cuidados ao paciente com os possíveis riscos decorrentes do uso do medicamento; o farmacêutico terá na farmacovigilância uma extensão desua prática, interagindo com a equipe, deacordo com a Organização M undial da Saúde ${ }^{6}$.

Estando nesse panorama de hospital de ensino e sentinela, o hospital em questão tem papel primordial na formação dos profissionais de saúde, por consequência no cenário da assistência à saúde. Sendo assim, faz-se necessário que o serviço de farmacovigilância atue no sentido de detectar, avaliar, compreender e prevenir efeitos adversos ou quaisquer problemas relacionados ao medicamento².

\section{Um panorama das prescrições no Brasil e no mundo}

Para Mário Borges Rosa, a maioria dos profissionais envolvidos com o problema (farmacêuticos, médicos e enfermeiros) não sabe identificar um erro de medicação e muitos só se dão conta de que houve o erro quando ele é grave, muitas vezes resultando em óbito. Os erros menores praticamente passam despercebidos e, em consequência disso, não são registrados, muito menos avaliados e estudados. Portanto, a prática profissional nas unidades de saúde deve ser 0 lugar para a discussão do assunto e implantação de medidas de prevenção de eventos adversos ${ }^{5}$.

Em um estudo realizado no ano de 2004 no Estado do Ceará, Neri concluiu que, apesar da existência de regulamentação brasileira para a prescrição em serviço público de saúde, que institui como obrigatório o uso da denominação genérica, o uso da denominação comercial ainda 
é elevado (ocorre em cerca de um terço dos itens prescritos) e que a forma adotada para redação das prescrições não satisfaz as condições de segurança necessárias ao processo de prescrição, 0 que constitui campo fértil para a omissão de elementos importantes quanto a garantir a segurança na dispensação e na administração do medicamento prescrito ${ }^{19}$.

A prescrição medicamentosa éum documento legal, pelo qual se responsabilizam quem prescreve (médico) equem dispensa o medicamento (farmacêutico) e está sujeito à legislação de controle e vigilância sanitários. No âmbito hospitalar, a prescrição de medicamento envolve, ainda, a equipe de enfermagem, que administra o medicamento ao paciente. Esse documento deveseguir normas legais e institucionais que definem como o fármaco deveser fornecido ao pacientee em que condições ele deve ser utilizado ${ }^{4}$.

No Brasil, a prescrição de medicamentos é normatizada pela Lei $n=5.991$, de 17 de dezembro de $1973^{21}$, que apresenta algumas normas para a execução adequada de uma receita. De acordo com a lei dos genéricos, no âmbito do SistemaÚ nico deSaúde(SUS), as prescrições pelo profissional responsável devem adotar obrigatoriamente a Denominação Comum Brasileira (DCB) ou, na sua falta, a Denominação Comum Internacional $(\mathrm{DCl})^{22}$.

Em 1996, Benet ${ }^{23}$ relata que "a prescrição médica é uma relação terapêutica importante entre médico e paciente, e representa o produto da perspicácia diagnóstica e da capacidade terapêutica do médico, fornecen do instruções destinadas ao alívio ou à restauração da saúde do paciente". Entretanto, mesmo a prescrição médica escrita dentro dos melhores padrões científicos atuais pode tornar-se inútil, se não for clara para o farmacêutico, e fornecendo instruções adequadas para o enfermeiro ou paciente sobre como preparar e administrar " ou utilizar " os medicamentos prescritos.

\section{Metodologia}

Foi feito um estudo observacional do tipo transversal, quantitativo, o qual foi aprovado pelo Comitê de Ética em Pesquisa da Universidade Federal do Rio Grande do N orte (UFRN). 0 estudo foi realizado no setor de farmácia do Hospital de Pediatria Prof. Heriberto Ferreira Bezerra-UFRN.

A partir da autorização do comitê, foram analisadas, durante três meses, todas as segun- das vias das prescrições dos pacientes hospitalizados. Para a análise, foi elaborado um formulário, no qual os dados foram tabulados. Foi feito um estudo piloto de um mês, no qual se padronizou a leitura das prescrições junto aos pesquisadores. Foram inclusas todas as prescrições dos pacientes internados nas enfermarias durante o período do estudo, excluindo-se as da enfermaria de oncologia, por ser esta recém-implantada, o que poderia causar al gum viés. Foi calculada a frequência simples dos dados utilizando a planilha eletrônica M S/Excel e, em seguida, foi realizada a análise descritiva. As variáveis observadas foram as seguintes: legi bilidade da prescrição, sendo considerada a prescrição ilegível quando apresentassemais detrês itensilegível epouco legível deum a três itens, nomenclatura do medicamento (nome genérico ou não), se o medicamento era padronizado ou não, presença dos elementos obrigatórios na prescrição (nome, registro, peso eleito do paciente; carimbo, assinatura do prescritor e data), além dos dados relativos ao medicamento (dose, via de administração, intervalo posológico e apresentação).

\section{Resultados ediscussão}

Após serem analisadas 1.590 prescrições, contendo, em média 4,47 medicamentos por prescrição, foram obtidos os dados a seguir.

Quanto à legibilidade das prescrições, deuma maneira geral, 64,47\% foram legíveis, 32,39\% foram pouco legíveis e 3,14\%, totalmente ilegíveis. Esse resultado é semelhante ao de outros estudos, como o deWinslow, Davidoff eBorum ${ }^{9}$, que encontrou $4 \%$ de prescrições ilegíveis, 0 de Néri ${ }^{19}, 3,29 \%$ e o de Carvalho e Vieira ${ }^{1}, 3,0 \%$. Dentre os itens analisados, os percentuais de ilegibilidade encontrados foram os seguintes: posologia (28,99\%), nome do prescritor $(25,53 \%)$, nome do paciente $(21,95 \%)$, peso $(9,87 \%)$, data $(6,48 \%)$ e eleito $(4,21 \%)$ (Figura 1$)$.

0 fato de apenas $64,47 \%$ das prescrições serem totalmente legíveis constitui um problema que deveser abordado e corrigido na instituição. A falta de legibilidade pode acarretar uma série de problemas, como a não identificação do prescritor, o que impossibilita a comunicação com ele e o aumento de horas na preparação das doses individuais dos pacientes, pois o auxiliar de farmácia terá que recorrer ao prescritor para saber o que foi prescrito ou ir ao posto de enfermagem para verificar as informações ilegíveis, com o consequente atraso na entrega da dose 
individual para ser administrada ao paciente.

O utro grave problema da ilegibilidade é a geração de interpretações equivocadas, levando à troca de medicamento, de paciente e/ou da via de administração, etc. Tudo isso pode ocorrer tanto na fase de dispensação quanto na de administração do medicamento. Tais erros podem ter consequências graves, até a própria morte do paciente. Portanto, énecessário que secriem mecanismos para evitar que as prescrições se apresentem ilegíveis. Uma boa al ternativa éa implantação da prescrição eletrônica. Porém, apenas essa medida não é suficiente; deve ser feito um trabaIho de conscientização dos prescritores, a fim de que passem a entender a importância da prescrição no processo de saúde.

Em relação aos elementos da prescrição, observa-se, na Figura 2, que a ausência do carimbo ocorreu na maioria das prescrições, o que dificulta a identificação do prescritor. 0 número do registro do paciente também esteve ausente, em $41,0 \%$, dificultando a identificação, principalmenteno caso de pacientes com nomes semelhantes. 0 peso esteve ausente em $19,56 \%$, impossibilitando ao farmacêutico conferir adequadamente a prescrição. A ausência dos elementos da prescrição dificulta a preparação, a conferência e a administração da dose do paciente. Sendo assim, éimprescindível que todos os elementos estejam presentes, para que seja prestada a melhor assistênciaao paciente.
Das 1.590 prescrições, $49,81 \%$ continham medicamentos prescritos por nome comercial, sendo que, do total de medicamentos prescritos (7.119), $18,65 \%$ o foram por nome comercial, o que está em desacordo com a Lei no 9.787, de 10 defevereiro de 199922, que diz que, nos serviços públicos, os medicamentos devem ser prescritos, obrigatoriamente, pelo nome genérico. Além disso, dificulta o ciclo do medicamento, pois, muitas vezes, o nome comercial prescrito difere do existenteno hospital, gerando dúvidas e risco de troca.

Em relação aos dados quanto à padronização, 19,69\% das prescrições continham medicamentos fora da padronização (Figura 3), perfazendo $5,25 \%$ do número total prescrito.

Dos medicamentos prescritos fora da padronização, os antiinflamatórios e os neurolépticos alcançaram um maior percentual, como se pode ver na Tabela 1. Esses dados demonstram que é necessário fazer uma revisão da padronização atual, bem como divulgá-la melhor entreos prescritores. Quando o medicamento é prescrito fora da padronização, pode ocorrer demora no início do tratamento ou o uso do medicamento nem ser iniciado, devido à dificuldade de aquisição no serviço público. Sendo assim, o ideal éque a padronização cubra a maioria das patologias atendidas no hospital e que seja amplamente divulgada, para que os prescritores possam saber com que arsenal terapêutico eles podem contar.

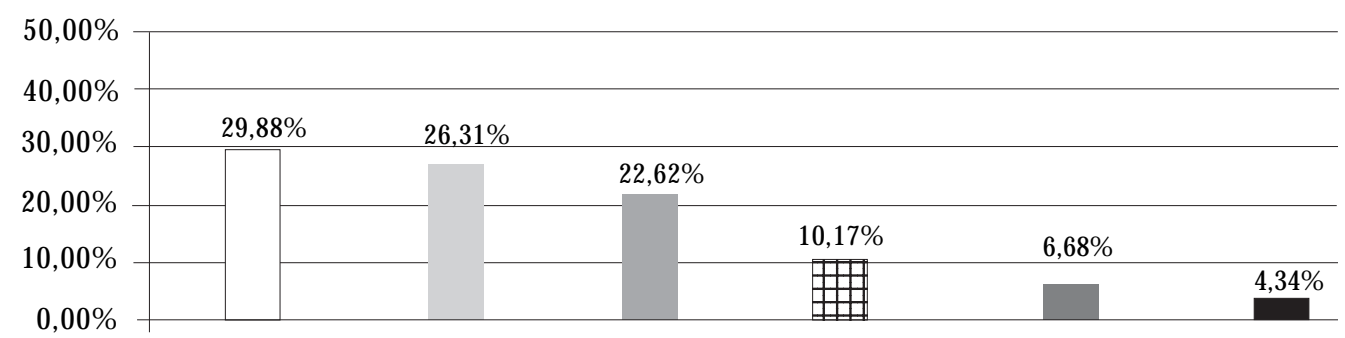

junho a agosto de 2007 - N atal, RN
Posologia
田 Leito
Nome do prescritor
Peso
Nome do paciente
Data

Figura 1. Distribuição percentual de legibilidade dos elementos da prescrição. 


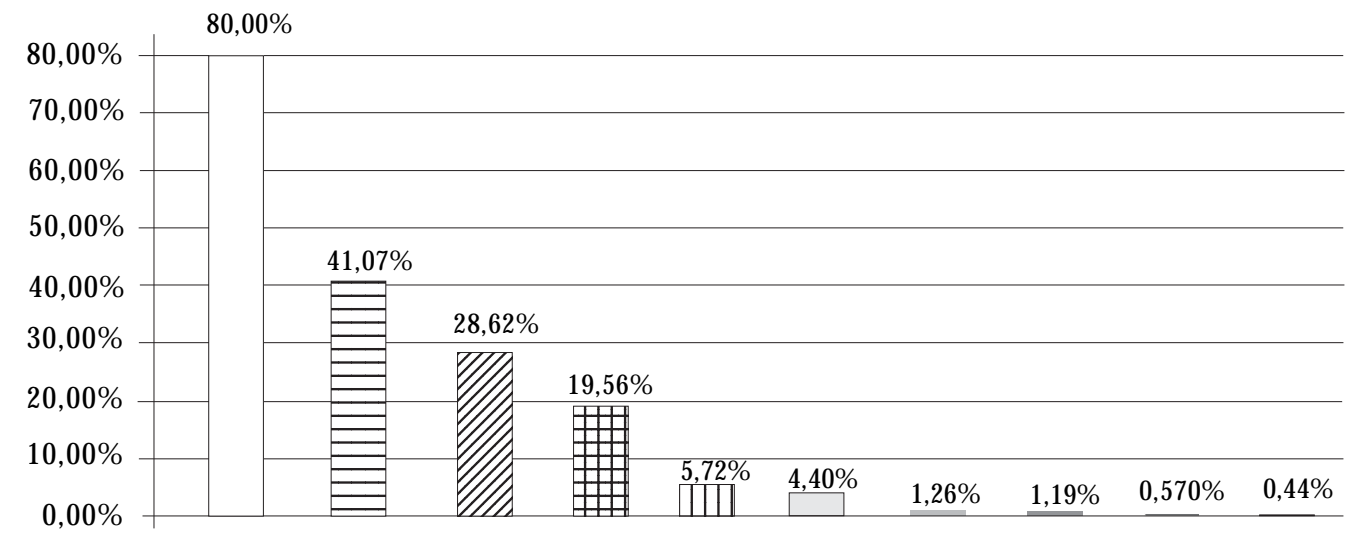

junho a agosto de 2007 - Natal, RN

\begin{tabular}{|ll|}
\hline Carimbo & $\square$ Via de administração \\
$\Xi$ Registro & Intervalo \\
$\square$ Apresentação & Dose \\
冊 Peso & Leito \\
$\square$ Assinatura & Nome do paciente \\
\hline
\end{tabular}

Figura 2. Distribuição percentual das prescrições, segundo a ausência de itens legais obrigatórios.

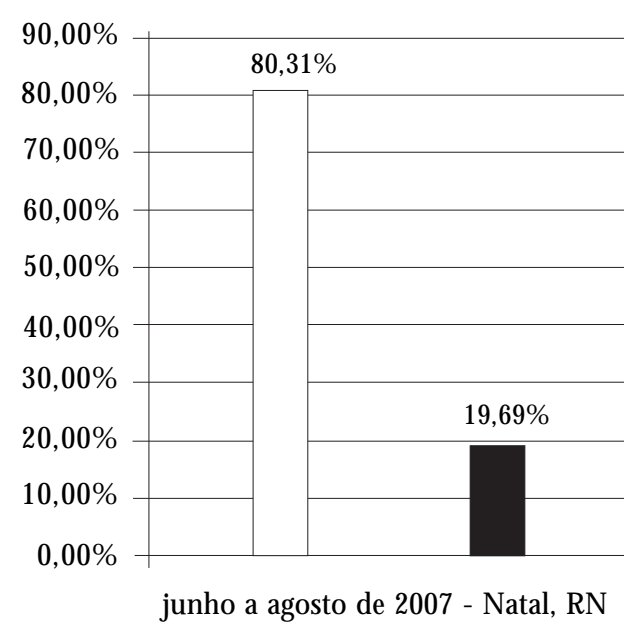

Prescrições contendo apenas medicamento padronizado

Prescirições contendo medicamento não padronizado

Figura 3. Distribuição da prescrição quanto à padronização dos medicamentos.
Tabela 1. Percentual de medicamentos prescritos fora da padronização.

\begin{tabular}{lr}
\hline \multicolumn{1}{c}{ M edicamentos não padronizados } & $\%$ \\
\hline Antiinflamatório & $15,65 \%$ \\
Neuroléptico & $14,78 \%$ \\
Enzima & $10,43 \%$ \\
Antiepiléptico & $9,57 \%$ \\
Eletrólitos & $7,83 \%$ \\
Corticóide & $7,83 \%$ \\
Antimicótico & $6,96 \%$ \\
Cáustico & $6,09 \%$ \\
Quimioterápico & $4,35 \%$ \\
Anticorpo & $4,35 \%$ \\
Antiácido & $2,61 \%$ \\
Cicatrizante & $2,61 \%$ \\
Anti-histamínico & $2,61 \%$ \\
Vitamina & $1,74 \%$ \\
Imunossupressor & $0,87 \%$ \\
Antisséptico & $0,87 \%$ \\
Antimicrobianos & $0,87 \%$ \\
\hline
\end{tabular}




\section{Considerações finais}

0 estudo realizado revela quea qualidade da prescrição do hospital em estudo precisa ser melhorada, a fim de que sejam evitados erros de medicação e o processo de assistência à saúde se torne mais seguro. A falta de clareza nas prescrições pode confundir os profissionais e causar danos ao paciente. Sendo assim, énecessário quesecriem mecanismos para tornar a prescrição o mais correta possível, atendendo ao que determinam a legislação e as normas institucionais. Para evitar erros relacionados à redação da prescrição médica, estudos apontam algumas medidas que podem ser tomadas. Uma delas tem se demonstrado mais efetiva, que é a implantação da prescrição eletrônica, a qual minimiza bastante os erros relacionados com a prescrição; porém, por si só, ela não elimina todos os erros, sendo ne- cessárias outras medidas, tai s como o treinamento dos prescritores " conscientizando-os e educando-os para a importância de uma prescrição correta ", a utilização de protocolos clínicos" que diminui a prescrição de medicamentos não padronizados; a padronização de processos e a expansão da atuação dos farmacêuticos clínicos.

A prescrição de $18,65 \%$ dos medicamentos pelo nome comercial demonstra que existe um forte apelo da indústria farmacêutica. Essa forma de prescrever está em desacordo com a legislação e pode causar erros de medicação, tendo em vista a semelhança de muitos nomes comerciais.

Além das medidas indicadas acima, é preciso mudar a visão em relação aos erros, procurando aceitá-los como evidência de falha no sistema, e encará-los como oportunidade de revisão e meIhoria dos processos, aprimorando, assim, a assistência prestada ao paciente.

\section{Colaboradores}

PT B Araújo eSAC U choa participaram igualmente de todas as etapas da elaboração do artigo.

\section{Agradecimentos}

A Evaldo Gonçalves de Araújo, Edileuza Gonçalves de Araújo, Flávia Karoline Jácome Quirino deO liveira eFillipe Azevedo de M edeiros. 


\section{Referências}

1. Carvalho RE, Cirilo HNC, Souza DF, Nogueira IAL, Effing C, Faleiro LC. Avaliação de fatores indutores a erros de dispensação em prescrições médicas do Hospital das Clínicas da UFG. Rev. SBRAFH 2007; 14.

2. Agência de Vigilância Sanitária. Farmacovigilância. [site da Internet] [acessado 2007 dez 07]. Disponível em: http://www.anvisa.gov.br/farmacovigilancia/ apresenta.htm

3. Fuchs FD, Wannmacher L, Ferreira M BC. Farmacologia clínica: Fundamentos da terapêutica racional. 3 a ed. Rio de Janeiro: Guanabara Koogan; 2004.

4. Portal da Saúde. U so racional de medicamentos. Conceito. [site da Internet] [acessado $2007 \mathrm{dez} 08$ ]. Disponível em: http://portal.saude.gov.br/saude/ visualizar_texto.cfm?dtxt $=25674$

5. Rosa M B. Anatomia dos erros. Entrevista. Rev. Pharmacia Brasileira 2005; 49.

6. Organização M undial da Saúde. The Uppsala M onitoring Centre. The Importance of Pharmacovigilance. Safety monitoring of medicinal products. Geneva: OM S; 2002.

7. Barros JAC. Políticas farmacêuticas: a serviço dos interesses da saúde? Brasília: UNESCO; 2004.

8. Cassiani SHB. Erros na medicação: estratégias de prevenção. Rev. Bras. Enf. 2000; 53(3):424-430.

9. Bobb A, Gleason K, Husch M, Feinglass J, Yarnold PR, Noskin GA. The epidemiology of prescribing errors: the potential impact of computerized prescribes order entry. Arch Intern M ed 2004; 164(7):785792.

10. Silva AEBC, Cassiani SHB. A prescrição de medicamentos como fonte de erros na medicação. Rev. Soc. Bras. Clin. M ed 2004; 2(6):157-164.

11. Lesar TS, Briceland L, Stein DS. Factors related to errors in medication prescribing. JAMA 1997; 277(4):312-317.

12. Ollivier V, Thelcide C, Simon C, Favier M. Standardized order form investigational drugs: effect on completeness of prescription. Pharm. World Sci 2004; 26(3):178-179.

13. Winslow EH, Nestor VA, Davidoff SK, Thompson PG, Borum JC. Legibility and completeness of physicians' handwritten medication order. Heart Hung 1997; 26(2):158- 164.

14. Yousif E, Ahmed AM, Abdalla ME, Abdelgadir MA. Deficiencies in medical prescriptions in Sudanese hospital. East M editer H ealth J 2006; 12(6): 915-918.
15. American Society of Healthy-System Pharmacists. Suggested definitions and relationships among medication misadventures, medication errors, adverse drug events, and adverse drug reactions. Am J H ealthSyst Pharm [periódico na Internet] 1998 [acessado 2007 ago 27];55:[cerca de 1 p.]. Disponível em: http:/ /www.nccmerp.org/pdf/tax02001-07-31.pdf

16. Rosa MB, Perini E. Erros de medicação: quem foi? Rev. Assoc. M ed. Bras. 2003; 49(3):335-341.

17. Brasil. Ministério da Saúde. Avaliação da assistência farmacêutica no Brasil: Estrutura, Processo e Resultado. Brasília: Ministério da Saúde; 2005.

18. Zangwill $A B$. Reducing prescribing errors through a quiz program for medical residents. Am J Health - Syst Pharm 2000; 57:1396-1397.

19. Neri EDR. Determinação do perfil dos erros de prescrição de medicamentos em um hospital universitário [dissertação]. Fortaleza (CE): Departamento de Farmácia, Faculdade de Farmácia, Odontologia e Enfermagem, Universidade Federal do Ceará; 2004.

20. M ello DR. Erros de medicação: o que diz o governo? Entrevista. Rev. Pharmacia Brasileira 2006; 51.

21. Brasil. Lei no 5.991, de 17 de dezembro de 1973. Dispõe sobre o controle sanitário do comércio de drogas, medicamentos, insumos farmacêuticos e correlatos e dá outras providências. Diário Oficial da União 1973; 19 dez.

22. Brasil. Lei no 9.787, de 10 de fevereiro de 1999. Dispõe sobre a vigilância sanitária estabelece o medicamento genérico, dispõe sobre a utilização de nomes genéricos em produtos farmacêuticos e dá outras providências. Diário O ficial da União 1999; $11 \mathrm{fev}$.

23. Benet LZ. Princípios utilizados na redação da receita médica e instruções a serem seguidas pelo paciente. In: H ardman JG, Limbrid LE. Goodman \& Giman's: as bases farmacológicas da terapêutica. 9a ed. São Paulo: McGraw-Hill; 1996. p. 1259-1265.

Artigo apresentado em 14/03/2008

Aprovado em 30/05/2008

Versão final apresentada em 06/08/2008 